\title{
Fases de la recuperación psicológica en pacientes dependientes del alcohol tras un tratamiento intensivo: Un seguimiento de 4 años
}

\section{Psychological recovery stages of alcohol dependent patients after an intensive outpatient treatment: A 4-year follow-up study}

\author{
Francisco Arias*,**,***, Ana Sion $* *$, Regina Espinosa****, Rosa Jurado-Barba**,****, \\ Marta Marín*, Andrés Martínez Maldonado**, Gabriel Rubio****,*****,******. \\ * Departamento de Psiquiatría. Hospital 12 de Octubre, Madrid. España. \\ ** Instituto de investigación biomédica. Hospital 12 de Octubre, Madrid. España. \\ *** Facultad de Psicología. Universidad Complutense de Madrid, Madrid. España. \\ **** Departamento de Psicología, Facultad de Educación y Ciencias de la Salud. Universidad Camilo José Cela, Madrid. España. \\ ***** Facultad de Medicina. Universidad Complutense de Madrid, Madrid. España. \\ ****** Red de Trastornos Adictivos. Instituto Carlos III, Madrid. España.
}

\section{Resumen}

El objetivo de este trabajo es comprobar si las recaídas dificultan la secuencia de la recuperación psicológica y reconstruir la secuencia de la recuperación de pacientes graves que solicitan tratamiento. Los participantes fueron 159 pacientes tratados durante dos años en un programa ambulatorio intensivo y tras ser dados de alta fueron seguidos durante cuatro años. En función de la presencia o no de recaída durante el seguimiento se configuraron dos grupos, el de abstinentes $(\mathrm{n}=80)$ y el de pacientes que recaen $(\mathrm{n}=79)$. Las evaluaciones se realizaron: basal, al alta del tratamiento, al $2 .^{\circ}$ y $4 .{ }^{\circ}$ año de seguimiento. Las variables fueron: conductas de evitación, ansiedad, depresión, impulsividad y sentido de la vida. Se incluyó un grupo de control $(\mathrm{n}=74)$ que fue evaluado con la misma cadencia que los pacientes. Los resultados indican una recuperación más lenta en el grupo con recaídas frente a los abstinentes, en todas las dimensiones psicológicas y los períodos estudiados. A los dos años de seguimiento, los pacientes abstinentes obtuvieron puntuaciones en depresión similares a los controles, además de puntuaciones superiores en sentido de la vida (MiL) a partir del final del tratamiento. Al menos en pacientes con dependencia grave del alcohol, nuestros resultados apoyan una secuencia de recuperación que podría continuar más allá de los cuatro años de seguimiento. Se inicia con la evitación de situaciones de riesgo y continúa con el resto de las dimensiones (ansiedad, depresión, impulsividad).

Palabras clave: Dependencia de alcohol; recuperación; sentido de la vida; abstinencia; sintomatología afectiva; impulsividad.

\begin{abstract}
The aim of this work is to determine if relapses can hinder the sequence of psychological recovery and to rebuild this sequence in patients with severe alcohol dependence that seek treatment. The sample was comprised of 159 patients seeking an intensive outpatient treatment of two years duration and who were subject to follow-up during four years after discharge. Patients were grouped according to the presence of relapse during follow-up, resulting in abstainers $(\mathrm{n}=80)$ and relapsers $(\mathrm{n}=79)$. Assessments were carried out in the following periods: baseline, at discharge, and at the second- and fourth-year follow-ups. The measurement variables were avoidance behavior, anxiety, depression, impulsivity and meaning in life (MiL). A control group $(\mathrm{n}=74)$ was evaluated at the same periods as the patients. Results indicate a slower recovery in relapsers in comparison to abstainers in all psychological dimensions and periods assessed. At the second-year follow-up, the abstainers achieved similar scores in depression as the control participants, in addition to higher scores in Meaning in Life at the end of treatment. In patients with severe alcohol dependence, our data supports a sequence of recovery that could continue beyond the four years of follow-up after treatment. This sequence would begin with the avoidance of risk situations and continue with the rest of dimensions (anxiety, depression, impulsivity). Keywords: Alcohol dependence; recovery; meaning in life; abstinence; affective symptoms; impulsivity.
\end{abstract}


$\mathbf{L}$ a Administración de Servicios de Salud Mental y Abuso de Sustancias (SAMHSA) (2011) define recuperación como «un proceso de cambio mediante el cual las personas mejoran su salud y bienestar, viven una vida autodirigida y se esfuerzan por alcanzar su máximo potencial» e incluye cuatro dimensiones importantes: salud, hogar, propósito y comunidad. La salud psicológica, que abarca los síntomas de la depresión y la ansiedad o el propósito de vida, ha sido una de las dimensiones más estudiadas relacionadas con la recuperación, aunque como predictor de la abstinencia o la recaída. De hecho, representan dimensiones que se han alterado durante el proceso de dependencia, estando asociadas a peores calidad de vida (Ugochukwu et al., 2013), bienestar psicológico y éxito del tratamiento en general (Amodeo, Kurtz y Cutter, 1992; Laudet, Becker y White, 2009). Específicamente, las personas con dependencia al alcohol presentan síntomas de ansiedad y/o depresión a lo largo del proceso de dependencia (Ghorbani, Khosravani, Bastan y Ardakani, 2017), así como una falta de motivación, problemas en la toma de decisiones, afrontamiento e impulsividad, entre otros (Ando et al., 2012; Brown, Vik, Patterson, Grant y Schuckit, 1995; Courtney et al., 2012). Se ha supuesto que con la abstinencia continuada comienza a producirse una normalización/ estabilización de estas variables (White, 2012); sin embargo, poco se sabe con respecto a la secuencia de recuperación y si los pacientes alguna vez alcanzan valores similares a la población sana (Kelly, Greene y Bergman, 2018).

Un aspecto importante de la recuperación podría ser la conducta de evitación, como una estrategia de afrontamiento con respecto a la exposición a sustancias mediante la evitación de situaciones de riesgo, es decir, donde el alcohol está presente o de contextos relacionados con el alcohol (p. ej., bares, fiestas, etc.). El afrontamiento de evitación es un elemento clave para los objetivos de prevención de recaídas, entre otras estrategias de afrontamiento (Marlatt, 1990; Marlatt y Witkiewitz, 2005), ya que tiene una presencia intensa desde las etapas iniciales del tratamiento psicoterapéutico.

Los estudios sobre la evolución de la ansiedad en pacientes con dependencia al alcohol que buscan tratamiento han determinado que tras varios meses de abstinencia se produce una disminución de sus puntuaciones en ansiedad; y que cuanto más altas son sus puntuaciones iniciales, mayor es el riesgo de recaída (Brown, Irwin y Schuckit, 1991; Rubio et al., 2017). Con respecto a los síntomas de depresión, la investigación también ha mostrado una reducción en las escalas de depresión a corto y largo plazo (Wilcox, Pearson y Tonigan, 2015; Worley, Tate y Brown, 2012). Estas puntuaciones más bajas en depresión se han asociado a un mayor nivel de asistencia a grupos de ayuda mutua, aunque su persistencia o presencia repentina e intensa supone un desafío en la recuperación de estos pacientes (Kelly, Stout, Magill, Tonigan y Pagano, 2010a). También se han observado niveles más bajos de impulsividad, al menos durante el primer año de abs- tinencia (Blonigen, Timko, Moos y Moos, 2009). Estudios realizados en sujetos con dependencia al alcohol que asistieron a grupos de ayuda mutua indican una disminución de la impulsividad de hasta quince meses de seguimiento (Blonigen, Timko, Finney, Moos y Moos, 2011; Kelly, Stout, Tonigan, Magill y Pagano, 2010b). Los resultados de nuestra investigación anterior también indican una mejora de estos síntomas, que podría durar más de dos años de tratamiento (Rubio et al., 2018).

$\mathrm{Al}$ mismo tiempo, la adquisición de valores como la espiritualidad y el sentido en la vida han sido consideradas como variables esenciales en la recuperación de la dependencia al alcohol, especialmente en estudios realizados en el campo de los programas basados en los 12 pasos, como Alcohólicos Anónimos (AA) (Tonigan, McCallion, Frohe y Pearson, 2017; Wilcox et al., 2015). La investigación de Kelly, Hoeppner, Stout y Pagano (2012) con dos muestras del proyecto MATCH mostró una correlación entre espiritualidad y asistencia a los grupos, particularmente en los pacientes incluidos en la muestra de atención continua $(n=774)$, es decir, los casos más graves. Otro valor implicado en la recuperación es el sentido de la vida que se ha relacionado con mejor funcionamiento social (Witkiewitz et al., 2019), calidad de vida (Laudet y White, 2008) y abstinencia continuada a largo plazo (Rubio et al., 2018). Un estudio reciente (Kelly et al., 2018) realizado en una muestra comunitaria de pacientes con dependencia al alcohol encontró que en los primeros meses de recuperación los índices de calidad de vida y bienestar psicológico eran inicialmente bajos, pero luego aumentaron, aunque no alcanzaron los niveles de la población general hasta 10 años después.

En resumen, hasta la fecha las investigaciones apuntan a una relación bidireccional entre duración de la abstinencia y mejora en la conducta de evitación, ansiedad, depresión, impulsividad, espiritualidad y propósito de vida. Sin embargo, no entendemos por completo cómo se produce la secuencia de recuperación psicológica y si es posible identificar diferentes etapas y duración. También faltan resultados sobre si esta recuperación, tras varios años de abstinencia, implicaría puntuaciones similares a los de la población general, respecto de las dimensiones psicológicas mencionadas.

De esta forma, el objetivo de este estudio fue determinar la evolución de las variables conductuales (evitación) y psicológicas (ansiedad, depresión, impulsividad y propósito de vida) durante el período de tratamiento intensivo de dos años de duración y cuatro años de seguimiento. En este estudio utilizamos una muestra de un estudio previo (Rubio et al., 2018) dividida por la presencia de recaída, formando dos grupos (recaídas y abstinentes), los cuales fueron objeto de seguimiento durante cuatro años. También incluimos un grupo de control formado por participantes de la población general.

Nuestra hipótesis es que la presencia de recaída ralentizaría el proceso de recuperación psicológica, y que incluso 
los abstinentes no alcanzarían puntuaciones similares a los participantes del grupo de control en las dimensiones psicológicas medidas (Kelly et al., 2018).

\section{Método}

\section{Participantes}

La muestra fue seleccionada durante un período de 14 meses entre pacientes asistentes al programa de tratamiento de dependencia al alcohol del Hospital 12 de Octubre (Rubio et al., 2018). Un total de 249 pacientes se incluyeron el estudio, con 42 abandonos en diferentes momentos del estudio (tercer $(\mathrm{N}=11)$, sexto $(\mathrm{N}=12)$, noveno $(\mathrm{N}=$ $9)$, duodécimo $(\mathrm{N}=4)$ y decimoctavo mes $(\mathrm{N}=5))$. Al final del seguimiento, teníamos información incompleta sobre 41 sujetos, ya sea porque fue difícil contactar con ellos durante el transcurso del tiempo o porque habían fallecido $(\mathrm{n}=7)$. La muestra final incluida en el análisis consistió de 233 sujetos. El grupo de pacientes se dividió según la presencia de recaídas durante el estudio, dando lugar a un primer grupo de abstinentes y un segundo grupo de recaídas. La recaída se define como el consumo de más de 4 unidades de bebidas estándar en hombres y 2,5 unidades en mujeres, durante al menos tres veces por semana o cantidades inferiores más de tres veces por semana.

Con respecto al grupo de control, los participantes fueron reclutados a través de anuncios colocados en dos centros de salud, solicitando la participación en un estudio para evaluar el progreso de los estados emocionales. Una vez entrevistados, se descartó a aquellas personas que cumplían criterios de abuso o trastornos por uso de sustancias, o cualquier otra afección psiquiátrica o neurológica. A los participantes se les explicaron las instrucciones de las pruebas y el procedimiento de seguimiento del estudio. La muestra inicial tenía 167 candidatos, pero 46 fueron descartados por abuso o trastornos por uso de sustancias y cuatro negaron participar en las medidas de seguimiento, mientras que 32 no asistieron al segundo año de evaluación de seguimiento. Se dispuso de datos completos de 85 casos, aunque la muestra final estuvo compuesta por 74 pacientes, ya que debían ser similares en edad y sexo a la muestra clínica.

La Tabla 1 muestra las características sociodemográficas y clínicas de todos los participantes. El $66 \%$ del total de la muestra eran hombres con una edad promedio de 42,86 $(8,35)$ años. El 59,9 \% había completado estudios secundarios, el 23,3\% estudios superiores y el 16,8 \% estudios de primaria. La mitad de la muestra tuvo un empleo durante la evaluación. En cuanto a los datos de consumo de alcohol, los pacientes refirieron niveles elevados de consumo diario y sus puntuaciones en EIDA (Rubio, Urosa y Santo Domingo, 1998) indicaron una dependencia grave del alcohol, con más de 10 años de evolución. Al examinar estas diferencias sociodemográficas entre los tres grupos, no encontramos diferencias estadísticamente significativas para la mayoría de las variables, con la excepción del nivel de estudios y los niveles de abstinencia en meses entre abstinentes y recaídas $(p<, 01$, ver Tabla 1$)$.

\section{Programas de tratamiento y entrevistas de seguimiento}

Los detalles sobre el programa terapéutico se pueden leer en Rubio et al. (2018). Los grupos clínicos fueron tratados de forma ambulatoria e intensiva, a lo largo de 24 meses, y se incluyeron de manera secuencial en los siguientes programas: desintoxicación y motivación para la abstinencia, prevención de recaídas, habilidades sociales, consolidación de hábitos y estilo de vida saludables y preparación para el alta. Anualmente, disponíamos de datos sobre la evolución de cada paciente. Después del alta, cada dos años se entrevistó a los participantes para completar escalas psicológicas, se les extraían muestras de sangre para determinar la GGT y se registró la información relativa a los meses de abstinencia y asistencia a las citas de seguimiento. La mitad de la muestra $(\mathrm{n}=91)$, además de asistir al programa ambulatorio, también participaba en grupos de ayuda mutua de la Federación de Alcohólicos de la Comunidad de Madrid (FACOMA), basado en el programa Ayúdate-Ayúdanos (FACOMA, 2016).

\section{Instrumentos de evaluación clínica}

Los pacientes fueron entrevistados y diagnosticados según los criterios del DSM-IV-TR (American Psychiatric Association, 2000). Se utilizó una versión en español (Rubio et al., 1998) del Cuestionario sobre la gravedad de la dependencia al alcohol (SADQ) (Stockwell, Murphy y Hodgson, 1983) para evaluar la intensidad de la dependencia al alcohol (EIDA), compuesto por 30 ítems tipo Likert con cuatro opciones de respuesta. Las puntuaciones totales en el EIDA pueden indicar dependencia baja $(<20)$, moderada (21-37) o grave (>37). Esta escala tiene buenos valores de fiabilidad, con valores de alfa de Cronbach de ,91 en la escala adaptada y de ,87 en este estudio. El seguimiento del consumo de alcohol se realizó a través de la entrevista Evaluación Retrospectiva del Consumo de Alcohol (TLFB) diseñada por Sobell, Sobell, Leo y Cancilla (1988) para determinar el consumo diario y la presencia de recaída, además del período de consumo y la abstinencia acumulada.

Las estrategias de afrontamiento de la dependencia al alcohol se evaluaron a través de una versión en español (García González y Alonso Suárez, 2002) del Inventario de Habilidades de Afrontamiento (CBI) (Litman, Stapleton, Oppenheim y Peleg, 1983). Esta medida autoinformada consta de 36 ítems y tiene como objetivo identificar la frecuencia de uso de estrategias de afrontamiento para mantener la abstinencia en situaciones de riesgo. Los valores de fiabilidad son de ,78 para la validación española y de ,76 en este estudio. Teniendo en cuenta que una de las estrategias menos utilizadas por los pacientes españoles es la evitación de la situación de riesgo, y su particular relevancia cultural 
Tabla 1. Características sociodemográficas y clínicas.

\begin{tabular}{|c|c|c|c|c|c|}
\hline & $\begin{array}{c}\text { Participantes } \\
n=233\end{array}$ & $\begin{array}{c}\text { Recaídas } \\
n=79\end{array}$ & $\begin{array}{c}\text { Abstinentes } \\
\mathbf{n}=\mathbf{8 0}\end{array}$ & $\begin{array}{c}\text { Controles } \\
n=74\end{array}$ & F. Welch/chi ${ }^{2}$ \\
\hline \multicolumn{6}{|l|}{ Sexo. no. (\%) } \\
\hline Hombres & $154(66,1)$ & $56(79,9)$ & $49(61,3)$ & $49(66,2)$ & 1,68 \\
\hline Edad. Media \pm SD & $42,8 \pm 8,35$ & $41,6 \pm 8,3$ & $43,4 \pm 8,4$ & $43,5 \pm 8,3$ & 1,24 \\
\hline \multicolumn{6}{|l|}{ Estado civil. N (\%) } \\
\hline Soltero/a & $56(35,2)$ & $30(38)$ & $26(32,5)$ & -- & \\
\hline Separado/a-Divorciado/a & $33(20,8)$ & $16(20,3)$ & $17(21,2)$ & -- & \\
\hline Viudo/a & $2(1,3)$ & $0(0)$ & $2(2,5)$ & -- & 2,37 \\
\hline \multicolumn{6}{|l|}{ Nivel de estudios. N (\%) } \\
\hline Primarios & $39(16,8)$ & $14(17,7)$ & $15(19)$ & $10(13,5)$ & \\
\hline Secundarios & $139(59,9)$ & $41(51,9)$ & $37(46,8)$ & $61(82,4)$ & \\
\hline Superiores & $54(23,13)$ & $24(30,4)$ & $27(34,2)$ & $3(4,1)$ & $27,59 \times$ \\
\hline \multicolumn{6}{|l|}{ Situación laboral. N (\%) } \\
\hline Desempleado/a & $32(20,1)$ & $21(26,6)$ & $11(13,8)$ & -- & \\
\hline ILT & $28(17,6)$ & $14(17,7)$ & $14(17,5)$ & -- & \\
\hline Pensionista & $11(6,9)$ & $5(6,3)$ & $6(7,5)$ & -- & 4,84 \\
\hline Ama/o de casa & $7(4,4)$ & $4(5,1)$ & $3(3,8)$ & -- & \\
\hline \multicolumn{6}{|l|}{ Grupo de tratamiento recibido. $n(\%)$} \\
\hline Regular & $68(29,2)$ & $46(58,2)$ & $22(27,5)$ & --- & \\
\hline Tipo mixto con FACOMA & $91(39,1)$ & $33(41,8)$ & $58(63,7)$ & --- & $15,33^{\star \star}$ \\
\hline \multicolumn{6}{|l|}{ Tipo de consumo. $\mathrm{N}(\%)$} \\
\hline Social & $33(21,2)$ & $16(20,8)$ & $17(21,5)$ & -- & \\
\hline Solitario & $47(30,1)$ & $27(35,1)$ & $20(25,3)$ & -- & \\
\hline Mixto & $76(48,7)$ & $34(44,2)$ & $42(53,2)$ & -- & 1,89 \\
\hline \multicolumn{6}{|l|}{ Frecuencia de uso. $\mathbf{N}(\%)$} \\
\hline Excesivo a diario & $134(84,8)$ & $69(88,5)$ & $65(81,2)$ & -- & \\
\hline Excesivo en fin de semana & $8(5,1)$ & $3(3,8)$ & $5(6,2)$ & -- & \\
\hline Excesivo esporádicamente & $16(10,1)$ & $6(7,7)$ & $10(12,5)$ & -- & 1,59 \\
\hline Vino & $10(6,3)$ & $4(5,1)$ & $6(7,5)$ & -- & \\
\hline Licores & $66(41,8)$ & $34(43,6)$ & $32(40)$ & -- & 0,48 \\
\hline Edad de inicio del consumo de alcohol. Media \pm SD & $17,24 \pm 5,35$ & $17,4 \pm 6,07$ & $17,9 \pm 6,4$ & $16,3 \pm 2,31$ & 3,07 \\
\hline Edad del diagnóstico de dependencia al alcohol Media \pm SD & $29,79 \pm 9,33$ & $29,8 \pm 9,31$ & $29,7 \pm 9,4$ & -- & 0,007 \\
\hline Años de consumo de alcohol. Media \pm SD & $12,82 \pm 10,07$ & $11,8 \pm 9,05$ & $13,8 \pm 10,9$ & -- & 1,54 \\
\hline Abstinencia en el primer año (en meses). Media \pm SD & $11,09 \pm 1,82$ & $10,6 \pm 2,2$ & $12 \pm 0$ & -- & $53,5^{\star \star}$ \\
\hline Abstinencia en el segundo año (en meses). Media \pm SD & $10,24 \pm 2,54$ & $8,46 \pm 2,59$ & $12 \pm 0$ & -- & $149,7^{\star \star}$ \\
\hline Abstinencia en el cuarto año (en meses). Media \pm SD & $9,14 \pm 3,4$ & $6,24 \pm 2,55$ & $12 \pm 0$ & -- & $407,1^{\star \star}$ \\
\hline \multicolumn{6}{|l|}{ Dependencia del tabaco. N (\%) } \\
\hline Sí & $129(81,2)$ & $61(77,2)$ & $66(82,5)$ & $21(29,6)$ & \\
\hline No & $20(12,6)$ & $12(15,2)$ & $8(10)$ & $50(70,4)$ & \\
\hline Abandono & $10(6,3)$ & $6(7,6)$ & $6(7,5)$ & $0(0)$ & $131^{\times x}$ \\
\hline \multicolumn{6}{|l|}{ Consumo de cocaína. N (\%) } \\
\hline No & $107(67,3)$ & $49(62)$ & $58(72,5)$ & -- & \multirow{3}{*}{2,01} \\
\hline Abuso & $22(13,8)$ & $13(16,5)$ & $9(11,2)$ & -- & \\
\hline Dependencia & $30(18,9)$ & $17(21,5)$ & $13(16,2)$ & -- & \\
\hline
\end{tabular}

Nota. Datos sociodemográficos y descriptivos clínicos e índices de comparación de valores estadísticos (ya sea Welch, $F$ y chi cuadrado) para los tres grupos de estudio (abstinentes, recaídas y grupo de control). ILT significa Incapacidad Laboral Temporal. «-» indica que estos datos no estaban disponibles en el caso del grupo de control. ${ }^{*},{ }^{\star *}$ indica valores $p$ de $<, 05$ y ,01, respectivamente.

en este país, decidimos incluir en este estudio los 5 ítems de la subescala de evitación (ítems 5, 8, 18, 20 y 30).

La sintomatología afectiva se evaluó mediante las escalas de ansiedad (HARS) (Hamilton, 1959) y de depresión (HDRS) de Hamilton (Hamilton, 1967), con valores alfa de Cronbach de ,78 y ,82, respectivamente, para este estudio. La impulsividad autoinformada se evaluó mediante la
Escala de Impulsividad de Barrat (BIS-11) (Patton, Stanford y Barratt, 1995), que consta de 30 ítems que evalúan la impulsividad cognitiva, motora y no planificada. La versión española (Oquendo et al., 2001) tiene un buen coeficiente alfa y mantiene la estructura de tres factores. En este estudio, el valor de alfa es de ,79. 
Otra medida utilizada en este estudio fue el Cuestionario de Significado de la Vida (MLQ), un instrumento diseñado para medir el sentido de la vida, es decir, el sentido de la propia naturaleza y existencia del sujeto (Steger, Frazier, Kaler y Oishi, 2006). Esta escala evalúa dos aspectos del sentido de la vida, a través de dos subescalas de cinco ítems: Presencia y Búsqueda. Presencia se refiere a la medida en que las personas comprenden, dan o perciben un sentido de su propia vida, junto con el grado de percepción de propósito, misión u objetivos. La versión española tiene un buen valor alfa de ,80 y en este estudio alcanza el valor de ,87. La validez concurrente muestra una buena asociación con el bienestar psicológico, donde el sentido de la vida se relacionaba con una vida comprometida y significativa (Góngora y Castro Solano, 2011).

\section{Procedimiento}

Se trata de un estudio de seguimiento de un grupo de pacientes que asisten a tratamiento por dependencia grave al alcohol, evaluados en cuatro ocasiones diferentes a lo largo de seis años: una evaluación basal (antes del tratamiento), al alta del tratamiento y a los dos y cuatro años después del tratamiento. También se evaluó un grupo de control en cuatro ocasiones cada dos años (evaluación basal, y a los dos, cuatro y seis años posteriores) para obtener una equivalencia con los períodos de evaluación de los grupos de pacientes.

\section{Análisis estadístico}

Las variables demográficas continuas se evaluaron mediante un análisis unilateral de varianza (ANOVA). Cuando las varianzas de las variables dependientes no eran iguales entre los grupos, usamos la prueba de Welch como una alternativa más robusta y conservadora que la prueba $\mathrm{F}$ habitual.

Utilizamos medidas repetidas en los ANOVA de dos por cuatro factores para afrontamiento de evitación, incorporando grupo (abstinentes y recaídas) x tiempo (basal, al alta, y seguimientos de dos y cuatro años). Utilizamos medidas repetidas en los ANOVA de tres por cuatro factores para las variables dependientes (i.e., ansiedad, depresión, impulsividad y MiL), incorporando grupo (abstinentes, recaídas y controles) X tiempo (basal, al alta, y seguimientos de dos y cuatro años). Los efectos principales y de interacción significativos se analizaron adicionalmente mediante comparaciones post-hoc con el nivel alfa ajustado con la corrección de Bonferroni. Todos los análisis estadísticos se realizaron con el paquete SPSS v.22 (IBM, 2013). Se realizó un análisis descriptivo y ANOVA adicionales para medidas repetidas para las subescalas de impulsividad, que se pueden consultar en el material complementario de este trabajo.

\section{Resultados}

La Tabla 2 y la Figura 1 muestran los datos de las medidas autoinformadas de los grupos clínico (abstinentes y

Tabla 2. Puntuaciones en la evaluación psicológica.

\begin{tabular}{|c|c|c|c|c|}
\hline Grupos & Inicio del estudio (línea basal) & Al alta & Seguimiento a 2 años & Seguimiento a 4 años \\
\hline \multicolumn{5}{|c|}{ Afrontamiento de evitación } \\
\hline Abstinentes $(n=80)$ & $6,60 \pm 1,51$ & $7,06 \pm 0,87$ & $6,85 \pm 0,91$ & $7,37 \pm 0,98$ \\
\hline Recaídas $(\mathrm{n}=79)$ & $5,37 \pm 1,64$ & $5,24 \pm 1,35$ & $5,18 \pm 1,14$ & $5,87 \pm 1,12$ \\
\hline \multicolumn{5}{|c|}{ Escala de ansiedad de Hamilton } \\
\hline Abstinentes $(n=80)$ & $11,31 \pm 5,51$ & $10,24 \pm 3,30$ & $9,19 \pm 1,61$ & $9,90 \pm 2,32$ \\
\hline Recaídas $(\mathrm{n}=79)$ & $11,4 \pm 5,63$ & $12,68 \pm 4,81$ & $10,03 \pm 6,85$ & $15,72 \pm 5,79$ \\
\hline Controles $(n=74)$ & $8,51 \pm 1,87$ & $8,38 \pm 1,88$ & $8,32 \pm 1,77$ & $8,05 \pm 2,08$ \\
\hline \multicolumn{5}{|c|}{ Escala de ansiedad de Hamilton } \\
\hline Abstinentes $(n=80)$ & $10,92 \pm 7,33$ & $8,03 \pm 3,64$ & $6,11 \pm 3,23$ & $5,55 \pm 2,82$ \\
\hline Recaídas $(\mathrm{n}=79)$ & $10,57 \pm 7,92$ & $9,76 \pm 4,60$ & $10,2 \pm 3,87$ & $11,3 \pm 3,11$ \\
\hline Controles $(n=74)$ & $5,97 \pm 2,04$ & $5,82 \pm 2,07$ & $5,89 \pm 2,07$ & $5,80 \pm 2,06$ \\
\hline Abstinentes $(n=80)$ & $49,5 \pm 14,45$ & $46,3 \pm 12,06$ & $42,7 \pm 9,96$ & $41,2 \pm 8,32$ \\
\hline Recaídas $(\mathrm{n}=79)$ & $57,7 \pm 10,27$ & $51,6 \pm 9,14$ & $48,4 \pm 6,14$ & $49,7 \pm 7,48$ \\
\hline Controles $(n=74)$ & $38,12 \pm 11,7$ & $37,2 \pm 11,73$ & $37,2 \pm 11,4$ & $36,7 \pm 11,17$ \\
\hline \multicolumn{5}{|c|}{ Cuestionario de Significado de la Vida } \\
\hline Abstinentes $(n=80)$ & $38,5 \pm 7,73$ & $47 \pm 6,52$ & $51,04 \pm 5,4$ & $58,4 \pm 5,95$ \\
\hline Recaídas $(\mathrm{n}=79)$ & $39,1 \pm 7,22$ & $41,7 \pm 6,85$ & $41,6 \pm 5,23$ & $46,3 \pm 6,05$ \\
\hline Controles $(n=74)$ & $44,3 \pm 7,13$ & $42,4 \pm 8,00$ & $44,1 \pm 8,04$ & $45,3 \pm 7,06$ \\
\hline
\end{tabular}

Nota. Datos descriptivos (media \pm SD) para medidas autoinformadas en los diferentes momentos de las evaluaciones (columnas), en cada grupo de estudio (filas). 

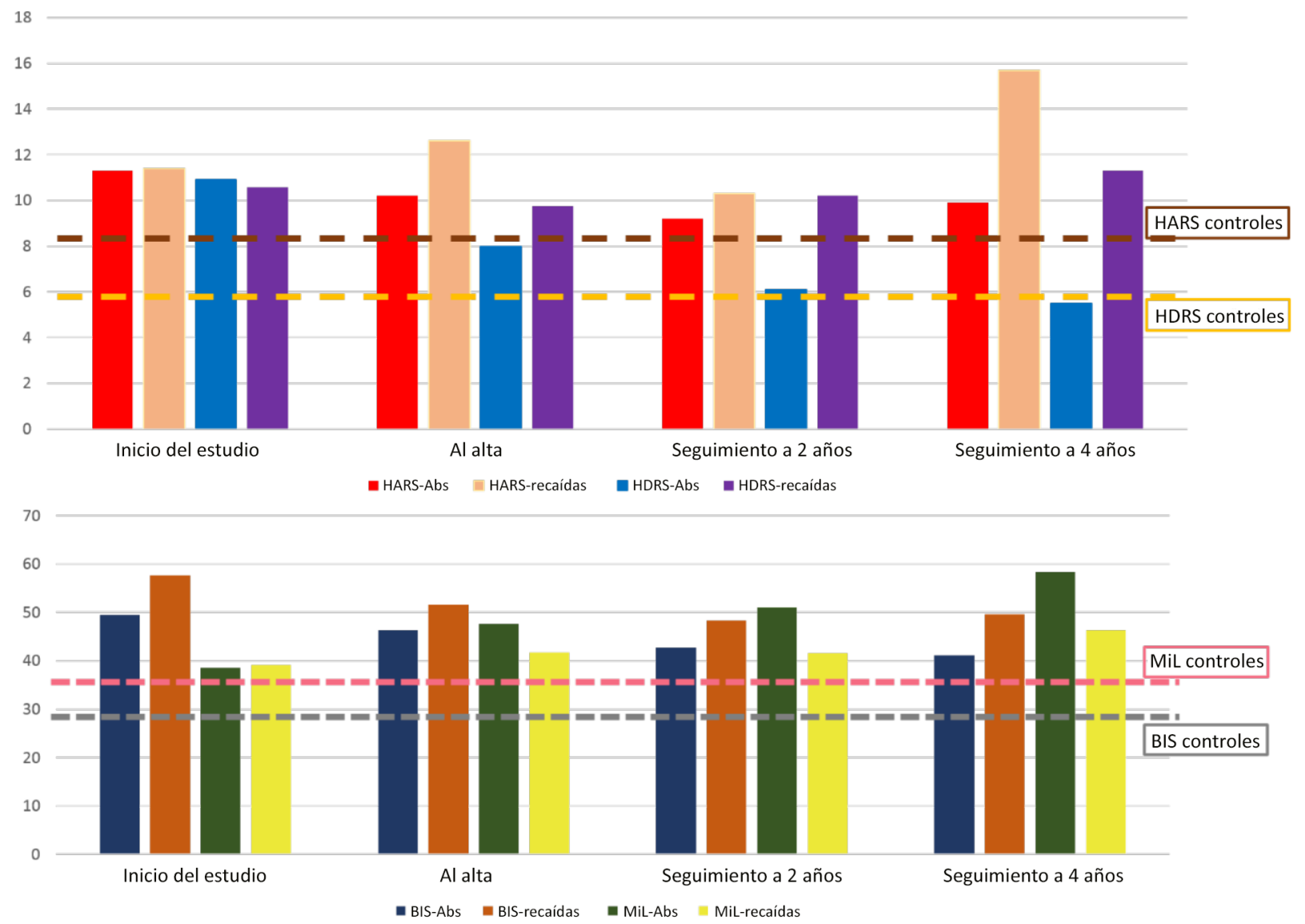

Figura 1. Cambios en los síntomas psicológicos.

Nota. Puntuaciones medias de ansiedad (HARS) y depresión (HDRS), impulsividad (BIS) y sentido de la vida (MiL) en varios momentos de la evaluación. Las columnas roja, azul, azul oscuro y verde representan puntuaciones medias para el grupo de abstinentes (Abs) en HARS, HDRS, BIS y MiL, respectivamente. Asimismo, los colores salmón, morado, marrón y amarillo representan las puntuaciones de las recaídas en las mismas medidas. En aras de la brevedad, representamos un valor promedio de estas medidas obtenido por los sujetos de control mediante el uso de líneas discontinuas (marrón oscuro, naranja, rosa y gris para HARS, HDRS, BIS y MiL, respectivamente).

recaídas) y de control, a lo largo de los diferentes períodos de evaluación.

\section{Afrontamiento de evitación durante el estudio}

Los resultados de esta escala solo se refieren a grupos de pacientes. Los abstinentes obtuvieron puntuaciones significativamente más altas en comparación con las recaídas ( $F$ = 166,44; $p=, 0001 ;$ et $a^{2}$ parcial $\left.=, 51\right)$. Este estudio también reveló un efecto significativo con respecto al momento de la medición: al final del estudio (al $4 .^{\circ}$ año de seguimiento), las puntuaciones fueron significativamente más altas tanto para los abstinentes como para las recaídas en comparación con los tres momentos previos de medición ( $\lambda$ de Wilk $=, 80 ; F=12,46 ; p=, 001 ;$ eta $^{2}$ parcial $\left.=, 19\right)$. En particular, en el grupo de abstinentes, las puntuaciones de afrontamiento de evitación aumentaron significativamente al alta y al cuarto año de seguimiento, en comparación con la línea de base y el segundo año de seguimiento. Para las recaídas, se encontraron diferencias significativas entre la línea basal, el cuarto año de seguimiento y las mediciones al alta, así como el segundo año de seguimiento ( $\lambda$ de Wilk $=, 97 ; F=1,55 ; p=, 20 ;$ et $a^{2}$ parcial $\left.=, 03\right)$.

\section{Recuperación de los síntomas de ansiedad}

Se observó un efecto significativo principal del factor grupo $\left(F=62,17 ; p=, 0001 ;\right.$ et $a^{2}$ parcial $\left.=0,35\right)$; los sujetos de control obtuvieron puntuaciones más bajas que los abstinentes y las recaídas. Este estudio también encontró un efecto principal significativo para el momento de la evaluación, revelando puntuaciones significativamente más bajas para el $2 .^{\circ}$ y $4 .^{\circ}$ año de seguimiento en comparación con los momentos basales y de alta ( $\lambda$ de Wilk $=, 72 ; F=28,89$; $p=, 001 ;$ et $a^{2}$ parcial $\left.=, 27\right)$. Se observó una disminución significativa de la ansiedad en el grupo de abstinentes al $2 .{ }^{\circ}$ año de seguimiento, aunque no se observaron diferencias al $4 .^{\circ}$ año. En cuanto al grupo de pacientes con recaída, los niveles de ansiedad aumentaron al alta, disminuyeron en el $2 .^{\circ}$ año de seguimiento y luego aumentaron de nuevo 
significativamente en el $4 .^{\circ}$ año de seguimiento. Los sujetos de control no mostraron diferencias significativas en las puntuaciones de ansiedad en los cuatro momentos de evaluación.

Por último, se observó un efecto de interacción entre grupo y momento de evaluación respecto a la ansiedad $(\lambda$ de Wilk $=, 65 ; F=17,97 ; p=, 0001 ;$ et $a^{2}$ parcial $\left.=, 19\right)$. Específicamente, al inicio del estudio, tanto las recaídas como los abstinentes mostraron niveles de ansiedad significativamente más altos en comparación con los participantes de control. Mientras que, al alta, el grupo de abstinentes mostró puntuaciones de ansiedad significativamente más bajas en comparación con las recaídas, aunque más altas en comparación con los participantes de control. Estos mismos resultados se observaron en el $2 .^{\circ}$ y $4 .^{\circ}$ año de seguimiento.

\section{Recuperación de síntomas depresivos}

De manera similar a los síntomas de ansiedad, el grupo de control mostró puntuaciones de depresión significativamente más bajas, seguido de los abstinentes y las recaídas $\left(F=43,1 ; p=, 0001 ;\right.$ et $a^{2}$ parcial $\left.=, 27\right)$. Se observan puntuaciones disminuidas de depresión desde las evaluaciones basales hasta el momento del alta, y se mantienen a lo largo de los seguimientos $\left(\lambda\right.$ de Wilk $=, 90 ; F=8,01 ; \mathrm{p}=, 001 ;$ et $^{2}$ parcial $=, 09)$. En el grupo de abstinentes, las puntuaciones alcanzadas hasta el 2. ${ }^{\circ}$ año de seguimiento se mantuvieron sin cambios significativos hasta el $4 .^{\circ}$ año de seguimiento, aunque fueron significativamente inferiores en comparación con los momentos basales y al alta. Las recaídas tuvieron niveles significativamente más bajos de depresión al alta, aunque aumentaron después de este momento. Los participantes de control mantuvieron niveles similares de depresión a lo largo del estudio. Se encontró una interacción significativa entre grupo y momento de evaluación $(\lambda$ de Wilk = ,78; $F=9,86 ; p=, 0001 ;$ et $a^{2}$ parcial $\left.=, 11\right)$, de tal manera que, al momento del alta, los abstinentes mostraron niveles significativamente menores de depresión en comparación con las recaídas, aunque mayor que los sujetos de control. En los $2 .^{\circ}$ y $4 .^{\circ}$ años de seguimiento, los abstinentes alcanzaron puntuaciones de depresión similares a los participantes de control y mantuvieron las puntuaciones más bajas en comparación con los pacientes con recaída.

\section{Recuperación de los síntomas de impulsividad}

En cuanto a la impulsividad, los sujetos de control obtuvieron las puntuaciones más bajas, seguido de los abstinentes y las recaídas $\left(F=41,1 ; p=, 0001 ;\right.$ eta $^{2}$ parcial $\left.=, 26\right)$. El momento de la evaluación también influyó en las puntuaciones $\left(\lambda\right.$ de Wilk $=, 59 ; F=52,87 ; p=, 001 ;$ et $a^{2}$ parcial $=$ ,41), de tal forma que los niveles de impulsividad al alta y dos años después del tratamiento fueron significativamente menores en comparación con la evaluación basal, y permaneció estable al $4 .^{\circ}$ año después del tratamiento. Especí- ficamente, los abstinentes descendieron significativamente en los niveles de impulsividad en todos los momentos de medición. Asimismo, las recaídas fueron reduciendo sus puntuaciones de impulsividad en todos los momentos, con la excepción del $4 .^{\circ}$ año, donde la impulsividad fue mayor en comparación con el 2. ${ }^{\circ}$ año de seguimiento. Con respecto a los sujetos de control, no tuvieron cambios en los niveles de impulsividad en los cuatro momentos. Por último, se observó un efecto de interacción significativo entre grupo y momento de evaluación respecto a la impulsividad ( $\lambda$ de Wilk $=, 67 ; F=16,42 ; p=, 0001 ;$ eta $a^{2}$ parcial $\left.=, 17\right)$. De esta forma, las recaídas mostraron niveles de impulsividad significativamente mayores en comparación con los abstinentes y los sujetos de control, en todos los momentos de evaluación. Asimismo, los abstinentes mostraron mayor impulsividad en comparación con los participantes de control en todos los momentos de evaluación.

\section{Sentido de la vida y recuperación}

Las puntuaciones en sentido de la vida (MiL) para los abstinentes fueron significativamente más elevadas en comparación con los otros dos grupos $(F=39,32 ; p=, 0001$; $e t a^{2}$ parcial $\left.=, 25\right)$. Se encontró un efecto principal significativo del momento de la evaluación, donde las puntuaciones de MiL fueron significativamente más altas en los momentos de seguimiento en el $2 .^{\circ}$ y $4 .^{\circ}$ año $(\lambda$ de Wilk $=$ , $50 ; F=74,14 ; p=, 001 ;$ et $^{2}$ parcial $\left.=, 49\right)$. Por un lado, los abstinentes tuvieron niveles significativamente crecientes de MiL durante el $2 .^{\circ}$ y $4 .^{\circ}$ año de seguimiento en comparación con momentos previos de evaluación. Las recaídas, por otro lado, mostraron importantes aumentos en MiL en todos los momentos, con la excepción del alta y el $2 .^{\circ}$ año después de los momentos de tratamiento, donde permanecieron estables. Por último, los niveles de MiL para los sujetos de control no mostraron diferencias entre los momentos de evaluación, a excepción de la diferencia encontrada entre la $2 .^{\mathrm{a}}$ y $4 .^{\mathrm{a}}$ evaluación, mostrando un incremento en la última.

Además, se observó un efecto de interacción entre grupo y momento de evaluación con respecto a las puntuaciones de MiL ( $\lambda$ de Wilk $=, 55 ; F=25,99 ; p=, 0001 ;$ et $^{2}$ parcial $=, 25)$. Las comparaciones post hoc muestran que, al inicio del estudio, ambos abstinentes y los pacientes con recaída tenían niveles de MiL significativamente más bajos en comparación con los sujetos de control. Mientras que, en el momento del alta, los abstinentes tenían puntuaciones MiL significativamente más altas en comparación con las recaídas y los participantes de control. Se mantuvieron resultados similares a los $2 .^{\circ}$ y $4^{\circ}$ años de seguimiento.

En resumen, los abstinentes mostraron niveles superiores de MiL después del tratamiento en comparación con los otros dos grupos de estudio y fueron aumentando progresivamente a lo largo de los momentos de seguimiento. 


\section{Discusión}

Los datos provenientes de este estudio pueden confirmar parcialmente la hipótesis inicial. Primero, el grupo con recaídas tuvo una recuperación más lenta en comparación con los pacientes abstinentes, en todas las dimensiones psicológicas evaluadas. No obstante, y contrariamente a nuestra hipótesis, los pacientes abstinentes alcanzaron puntuaciones similares a los participantes de control en la escala de depresión, al 2. ${ }^{\circ}$ año de seguimiento. También mostraron puntuaciones más altas en sentido en la vida en comparación con los controles, desde el momento del alta del tratamiento en adelante. Además, con respecto a las etapas de la secuencia de recuperación psicológica, al analizar la situación de los abstinentes, observamos que la conducta de evitación se mantuvo a lo largo del tratamiento y los seguimientos; que las dimensiones que dependían menos de la personalidad, como los síntomas depresivos, se mantuvieron estables e incluso fueron similares a los sujetos de control dos años después del tratamiento; mientras que las variables más relacionadas con la personalidad, como la ansiedad y la impulsividad, continuaron disminuyendo durante el período de seguimiento, aunque con puntuaciones más altas que los individuos de control. En este grupo, el sentido de la vida se incrementó después de finalizar el tratamiento, con puntuaciones superiores incluso para los participantes de control.

\section{¿Por qué las recaídas suponen una ralentización del proceso de recuperación?}

Desde un punto de vista neurobiológico, las recaídas o interrupción de la abstinencia suponen una reactivación de los procesos de daños neurotóxicos (Crews, 2008), activación del estrés (Beracochea, Mons y David, 2019), los procesos neuroinflamatorios (Crews et al., 2005; Venner et al., 2006) e interrupción de la neurogénesis (Crews y Nixon, 2009), dando lugar a un empeoramiento de los síntomas de ansiedad (Brown et al., 1991; Rubio et al., 2017) y depresión (Kelly et al., 2010a).

Las recaídas suelen afectar el estado psicológico del paciente (Marlatt y Gordon, 1985), un impacto que podría prolongarse de forma significativa en el tiempo. Regularmente conducen a una rápida aparición de emociones negativas, como culpa y vergüenza, que aumentan la sensación de ineficacia y facilitan el proceso de recaída, con la consecuente exacerbación de los síntomas de ansiedad y depresión (de Hooge, Zeelenberg y Breugelmans, 2010).

\section{Los pacientes no alcanzaron puntuaciones similares a los controles en las dimensiones psicológicas evalua- das}

Como planteó la hipótesis, los pacientes de ambos grupos tenían niveles más altos de ansiedad e impulsividad en comparación con los participantes de control, a lo largo de todas las evaluaciones. Nuestros hallazgos son coheren- tes con los publicados por Kelly et al. (2018), aunque las dimensiones evaluadas no fueron del todo iguales. En su estudio, los pacientes en recuperación tardaron un promedio de 10 años en alcanzar niveles de calidad de vida similares a los de la muestra de control. Dado que la calidad de vida no es lo mismo que la ansiedad o la impulsividad, podríamos pensar que estas puntuaciones más altas tienen varios orígenes: se ha demostrado que las puntuaciones altas en ansiedad o impulsividad son un factor de riesgo para el desarrollo de dependencia de sustancias en la adolescencia (Chow et al., 2018; Dyer, Heron, Hickman y Munafó, 2019; Stautz y Cooper, 2013), aunque también pueden resultar de los efectos neurotóxicos del alcohol (Beracochea et al., 2019; Mons y Beracochea, 2016). Por tanto, las diferencias en ansiedad e impulsividad con el grupo de control podrían deberse a una combinación de factores de personalidad, previos al desarrollo de la dependencia al alcohol y también podrían surgir de los efectos dañinos del alcohol.

Algo diferente sucedió con las puntuaciones en depresión y sentido de la vida para los abstinentes. La recuperación de estas variables parece relacionarse más con la duración de la abstinencia y la asistencia a grupos de ayuda mutua (Kelly et al., 2010a). Por tanto, la normalización de las puntuaciones en depresión o MiL para los abstinentes podría explicarse por su mayor asistencia a los grupos de ayuda mutua (Kelly et al., 2012; Kelly et al., 2010a).

El hecho de que fueran necesarios varios años de abstinencia para lograr niveles normalizados respecto de la depresión o para impulsar el sentido en la vida, concuerda con las opiniones que tienen los pacientes que asisten a grupos de ayuda mutua (FACOMA, 2016). Otros modelos que exploran la recuperación de las dimensiones biopsicosociales también coinciden con la necesidad de que transcurran varios años para lograr cambios de personalidad que permitirían la adaptación a un nuevo estilo de vida (Chapman, 1991; Freyer-Rose, 1991; Gorski, 1990).

\section{¿Se puede establecer una secuencia de recuperación psicológica en la dependencia grave al alcohol?}

Si pensamos en el grupo de abstinentes como un modelo de recuperación de dependencia grave, donde se ha eliminado el impacto de la recaída, nuestros resultados permitirían plantear una hipótesis respecto de una secuencia de algunas variables psicológicas representadas en la Figura 2. Los cambios conductuales (afrontamiento de evitación) iniciados de manera significativa en los momentos iniciales del tratamiento son aquellos que permitirían un entorno seguro, indispensable para evitar recaídas y seguir introduciendo nuevos hábitos saludables.

Posiblemente, la percepción de la autoeficacia en la evitación de recaídas podría facilitar la gestión de emociones como la ansiedad, la depresión y el control del comportamiento impulsivo. Dado que muchas de estas dimensiones están estrechamente relacionadas con los factores de 


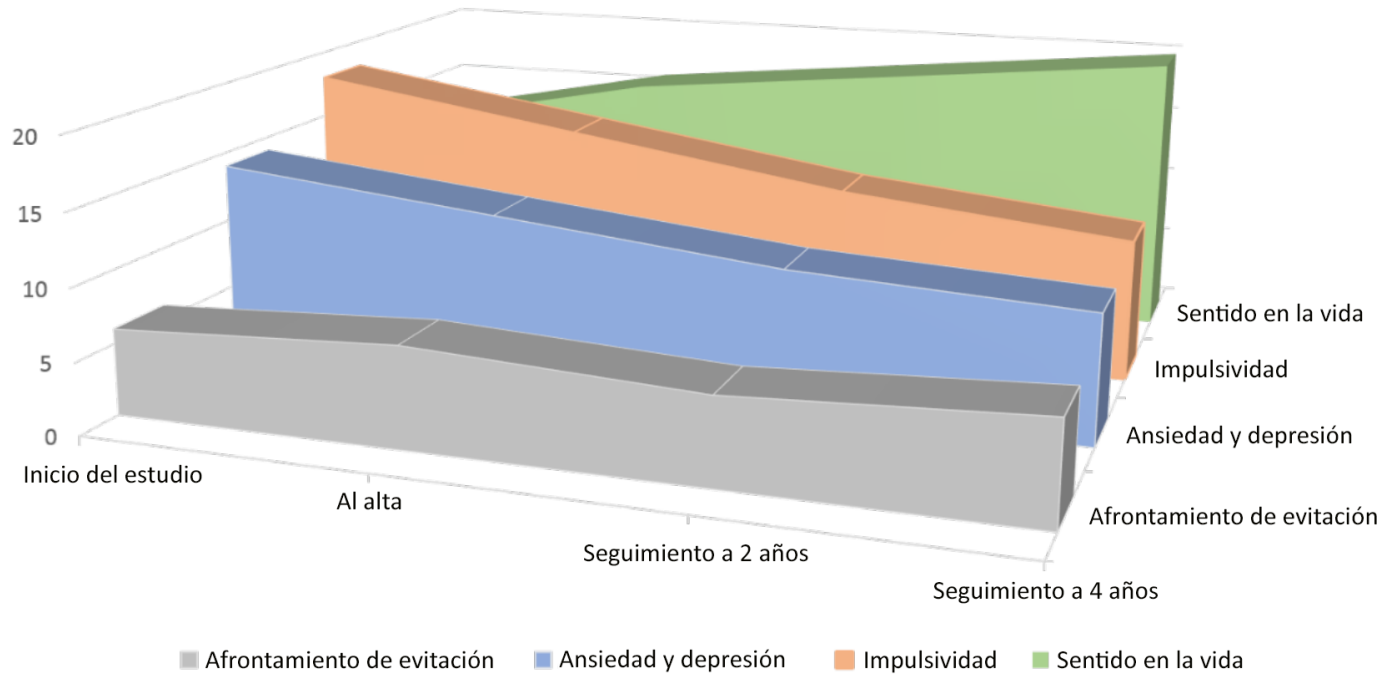

Figura 2. Etapas de recuperación psicológica a lo largo del estudio: conductual-emocional-sentido en la vida.

Nota. Una representación visual de la secuencia de recuperación psicológica en cada momento de la evaluación (eje X): inicio del estudio, al alta del tratamiento, y seguimientos a 2 y 4 años. El eje Y representaría cambios teóricos a lo largo de estos períodos en varias dimensiones psicológicas (eje Z) (sentido en la vida, impulsividad, ansiedad y depresión y afrontamiento por evitación).

personalidad y con una larga lista de hábitos aprendidos, sería plausible suponer que tardaría meses o incluso años para que los pacientes mejoren sus puntuaciones en estas dimensiones. Con el tiempo, los individuos serían capaces de cambiar su motivación para mantener la abstinencia, de modo que las motivaciones externas (preocuparse por las consecuencias en la salud física y psicológica relacionadas con el consumo de alcohol, así como los ámbitos laboral y familiar) son reemplazadas por motivaciones internas (coherencia, sentido en la vida, sentido de pertenencia a los grupos de ayuda mutua). Esta hipótesis es coherente con los enfoques motivacionales basados en el mantenimiento de los cambios conductuales (Kwasnicka, Dombrovski, White y Sniehotta, 2016).

Empíricamente, sí se han realizado estudios científicos rigurosos sobre cómo exactamente los grupos de ayuda mutua, como AA o FACOMA, benefician la recuperación. Estos estudios sugieren que las principales formas en que AA es útil para la remisión y la recuperación es facilitando cambios en las redes sociales de los asistentes y aumentando la autoeficacia de la abstinencia, el afrontamiento y manteniendo la motivación de la abstinencia (Kelly et al., 2010a; Kelly et al., 2010b). La evidencia también sugiere que estos amplios beneficios pueden depender de la gravedad de la dependencia (Kelly et al., 2012) y también del género (Kelly y Hoeppner, 2013), por lo que para las personas con adicciones más graves, además de facilitar importantes cambios en sus redes sociales, AA también puede ayudar a la recuperación reduciendo el afecto negativo y aumentando las prácticas espirituales. También es posible que MiL pueda ser una variable determinante, incluso desde los momentos iniciales del tratamiento, al favorecer la abstinencia al aumentar las estrategias de evitación y disminuir los niveles de depresión.

En cuanto a las limitaciones de este estudio, es posible que nuestros resultados no sean aplicables a una dependencia leve, ni a individuos que no buscan tratamiento, ya que este estudio incluyó pacientes con dependencia grave al alcohol que buscan tratamiento. Otra limitación podría representarse por el hecho de que un porcentaje importante de pacientes asistió a grupos de terapia de ayuda mutua. Esto podría dar lugar a diferencias entre sujetos en varias dimensiones psicológicas y aspectos de la recuperación. De hecho, ya se había abordado esta cuestión (Rubio et al., 2017) y se encontró que los pacientes que asisten a grupos de autoayuda FACOMA, además de los tratamientos psicoterapéuticos, mejoraron en síntomas afectivos y sentido de la vida, acumulando más meses de abstinencia.

Todos los pacientes contaban con apoyo familiar, siendo uno de los requisitos para entrar al estudio, por lo que ignoramos si el curso de las variables estudiadas hubiera sido el mismo en condiciones de apoyo distintas. La abstinencia se registró en base a informes autoinformados, por lo que no se pudo asegurar una ausencia total de consumo que los pacientes podrían haber ocultado; aunque también es cierto que los autoinformes suelen tener una buena correlación con las cantidades reales de consumo. Dado el considerable número de pacientes que no pudo completar estas medidas, podríamos plantear la hipótesis de que los cambios observados en este estudio corresponderían al grupo con mejores resultados. Aunque esta es una posibilidad, al menos disponemos de una secuencia de recuperación en un grupo de pacientes, aunque menos vulnerables a la recaída. 
Por último, una limitación adicional podría estar constituida por el hecho de que no disponemos del estado civil ni la situación laboral de los sujetos de control. Si bien este tipo de información sociodemográfica podría ser útil respecto a su posible impacto sobre varias variables psicológicas, no fueron la principal preocupación de los objetivos de este estudio y la investigación adicional sobre este tema debería tener en cuenta estas variables.

Implicaciones de este estudio: dado que las dimensiones psicológicas de impulsividad y ansiedad estudiadas en este trabajo no se estabilizaron hasta los 2+2 años de abstinencia, creemos importante subrayar la necesidad de seguimientos por parte de los equipos de atención primaria, para impulsar la revisión de pacientes en recuperación.

Pensamos que sería importante recomendar o insistir en la conveniencia de asistir a grupos de ayuda mutua como estrategia de utilidad para mejorar los estados emocionales relacionados con la ansiedad, la depresión y la impulsividad, y lo que es más importante, para engendrar o potenciar valores como el sentido en la vida (Kelly y Yeterian, 2013; Rubio et al., 2018). Desde nuestro punto de vista, la recuperación en pacientes graves comenzaría con un componente conductual (cambios en el estilo de vida), seguido de uno emocional (ansiedad y depresión) y un paso final basado en el propósito de vida y la espiritualidad.

\section{Reconocimientos}

La contribución de GR al estudio recibió apoyo del Instituto de Salud Carlos III (número de beca: FIS15-00463). Además, las aportaciones de RJB y RE contó con el apoyo de la Universidad Camilo José Cela (número de beca: 2018-02).

Nuestro agradecimiento a la Federación de Asociaciones de Alcohólicos y Familiares de la Comunidad de Madrid, FACOMA-Red C.A.P.A.

\section{Conflicto de intereses}

Los autores declaran la inexistencia de conflicto con entidades públicas o privadas.

\section{Referencias}

American Psychiatric Association (2000). DSM-IV-TR: diagnostic and statistical manual of mental disorders (4th ed). Washington, DC: American Psychiatric Association. doi:10.1007/978-3-642-28753-4_1094.

Amodeo, M., Kurtz, N. y Cutter, H. S. (1992). Abstinence, reasons for not drinking, and life satisfaction. The International Journal of the Addictions, 27, 707-716. doi:10.3109/10826089209068762.

Ando, B., Must, A., Kurgyis, E., Szkaliczki, A., Drotos, G., Rozsa, S., ... Almos, P. Z. (2012). Personality traits and coping compensate for disadvantageous decision-making in long-term alcohol abstinence. Alcohol and Alcoholism, 47, 18-24. doi:10.1093/alcalc/agr144.

Beracochea, D., Mons, N. y David, V. (2019). Targeting the glucocorticoid receptors during alcohol withdrawal to reduce protracted neurocognitive disorders. Frontiers in Psychiatry, 10, 580. doi: 10.3389/fpsyt.2019.00580.

Blonigen, D. M., Timko, C., Finney, J. W., Moos, B. S. y Moos, R.H. (2011). Alcoholics Anonymous attendance, decreases in impulsivity and drinking and psychosocial outcomes over 16 years: Moderated-mediation from a developmental perspective. Addiction, 106, 2167-77. doi:10.1111/j.1360-0443.2011.03522.x.

Blonigen, D. M., Timko, C., Moos, B. S. y Moos, R. H. (2009). Treatment, Alcoholics Anonymous, and 16year changes in impulsivity and legal problems among men and women with alcohol use disorders. Journal of Studies on Alcohol and Drugs, 70, 714-725. doi:10.15288/ jsad.2009.70.714.

Brown, S. A., Irwin, M. y Schuckit, M. A. (1991). Changes in anxiety among abstinent male alcoholics. Journal of Studies on Alcohol, 52, 55-61. doi:10.15288/jsa.1991.52.55.

Brown, S. A., Vik, P. W., Patterson, T. L., Grant, I. y Schuckit, M. A. (1995). Stress, vulnerability and adult alcohol relapse. Journal of Studies on Alcohol, 56, 538-545. doi:10.15288/jsa.1995.56.538.

Chapman, R. (1991). Middle recovery: An introspective journey. Addiction E Recovery, Sept-October, 8-12.

Chow, P. I., Portnow, S., Zhang, D., Salemink, E., Wiers, R. W. y Teachman, B. A. (2018). Comorbid interpretation and expectancy bias in social anxiety and alcohol use. Anxiety, Stress and Coping, 31, 669-685. doi:10.1080/1061 5806.2018.1521958.

Courtney, K. E., Arellano, R., Barkley-Levenson, E., Galvan, A., Poldrack, R. A., Mackillop, J., Jentsch, J. D. y Ray, L. A. (2012). The relationship between measures of impulsivity and alcohol misuse: An integrative structural equation modeling approach. Alcoholism: Clinical and Experimental Research, 36, 923-931. doi:10.1111/j.15300277.2011.01635.x.

Crews, F. T. (2008). Alcohol-related neurodegeneration and recovery: Mechanisms from animal models. Alcohol Research Eं Health, 31, 377-388. doi:10.1093/alcalc/ agn079.

Crews, F. T., Buckley, T., Dodd, P. R., Ende, G., Foley, N., Harper, C., ... Sullivan, E. V. (2005). Alcoholic neurobiology: Changes in dependence and recovery. Alcoholism: Clinical and Experimental Research, 29, 1504-1513. doi:10.1097/01.alc.0000175013.50644.61.

Crews, F. T. y Nixon, K. (2009). Mechanisms of neurodegeneration and regeneration in alcoholism. Alcohol and Alcoholism, 44, 115-127. doi:10.1093/alcalc/agn079.

Dyer, M. L., Heron, J., Hickman, M. y Munafò, M.R. (2019). Alcohol use in late adolescence and early adulthood: 
The role of generalized anxiety disorder and drinking to cope motives. Drug and Alcohol Dependence, 204, 107480. doi:10.1016/j.drugalcdep.2019.04.044.

Federación de Alcoholicos de la Comunidad de Madrid. (2016). Ayúdate-Ayúdanos.Programa para pacientes y familiares de personas con problemas por el alcohol u otras drogas. Madrid: Ediciones Mapa. ISBN 978-84-608-9611-1.

Freyer-Rose, K. (1991). Late recovery: A process of integration. Addiction E Recovery, Nov-December, 20-23.

García González, R. y Alonso Suárez, M. (2002). Evaluación en programas de prevención de recaída: Adaptación española del inventario de habilidades de afrontamiento (cbi) de litman en dependientes del alcohol. Adicciones, 14, 455-463. doi:10.1080/02791072.2002.10399968.

Ghorbani, F., Khosravani V., Bastan, F. S. y Ardakani, R. J. (2017). The alexithymia, emotion regulation, emotion regulation difficulties, positive and negative affects, and suicidal risk in alcohol-dependent outpatients. Psychiatry Research, 252, 223-230. doi: 10.1016/j.psychres.2017.03.005.

Góngora, V. y Castro Solano, A. (2011). Validación del cuestionario de significado de la vida mlq en población adulta y adolescente argentina. Interamerican Journal of Psychology, 45, 395-404.

Gorski, T. T. (1990). The cenaps model of relapse prevention: Basic principles and procedures. Journal of Psychoactive Drugs, 22, 125-133. doi:10.1080/02791072.1990.104 72538 .

Hamilton, M. (1959). The assesment of anxiety states by rating. British Journal of Medical Psychology, 32, 50-55. doi:10.1111/j.2044-8341.1959.tb00467.x.

Hamilton, M. (1967). Development of a rating scale for primary depressive illness. British Journal of Social and Clinical Psychology, 6, 278-296. doi:10.1111/j.2044-8260.1967. tb00530.x.

de Hooge, I. E., Zeelenberg, M. y Breugelmans, S. M. (2010). Restore and protect motivations following shame. Cognition and Emotion, 24, 111-127. doi:10.1080/02699930802584466.

IBM Corporation (2013). IBM SPSS Statistics for Windows, Version 22.0. Armonk, New York: IBM Corporation.

Kelly, J. F. y Yeterian, J. (2013). Mutual-help groups for alcohol and other substance use disorders. En McCrad, B. S. y Epstein, E. E. (Eds.). Addictions: A comprehensive guidebook (p. 500-525). New York: Oxford University Press.

Kelly, J. F., Greene, M. C. y Bergman, B. G. (2018). Beyond abstinence: Changes in indices of quality of life with time in recovery in a nationally representative sample of u.s. Adults. Alcoholism: Clinical and Experimental Research, 42, 770-780. doi:10.1111/acer.13604.

Kelly, J. F., Hoeppner, B., Stout, R. L. y Pagano, M. (2012). Determining the relative importance of the mechanisms of behavior change within alcoholics anonymous:
A multiple mediator analysis. Addiction, 107, 289-99. doi:10.1111/j.1360-0443.2011.03593.x.

Kelly, J. F. y Hoeppner, B. B. (2013). Does alcoholics anonymous work differently for men and women? A moderated multiple-mediation analysis in a large clinical sample. Drug and Alcohol Dependence, 130, 186-193. doi:10.1016/j.drugalcdep.2012.11.005.

Kelly, J. F., Stout, R. L., Magill, M., Tonigan, J. S. y Pagano, M. E. (2010a). Mechanisms of behavior change in alcoholics anonymous: Does alcoholics anonymous lead to better alcohol use outcomes by reducing depression symptoms? Addiction, 105, 626-636. doi:10.1111/j.13600443.2009.02820.x.

Kelly, J. F., Stout, R. L., Tonigan, J. S., Magill, M. y Pagano, M. E. (2010b). Negative affect, relapse, and alcoholics anonymous (aa): does aa work by reducing anger? Journal of Studies on Alcohol and Drugs, 71, 434-444 doi:10.15288/jsad.2010.71.434.

Kwasnicka, D., Dombrowski S. U., White, M. y Sniehotta, F. (2016). Theoretical explanations for maintenance of behaviour change: A systematic review of behaviour theories. Health Psychology Review, 10, 277-296. doi:10.10 80/17437199.2016.1151372.

Laudet, A. B., Becker, B., J. y White, W. L. (2009). Don't wanna go through that madness no more: Quality of life satisfaction as predictor of sustained remission from illicit drug misuse. Substance Use E् Misuse, 44, 227-252. doi:10.1080/17437199.2016.1151372.

Laudet, A. B. y White, W. L. (2008). Recovery capital as prospective predictor of sustained recovery, life satisfaction, and stress among former poly-substance users. Substance Use and Misuse, 43, 27-54. doi:10.1080/10826080701681473.

Litman, G. K., Stapleton, J., Oppenheim, A. N. y Peleg, B. M. (1983). An instrument for measuring coping behaviours in hospitalized alcoholics: Implications for relapse prevention treatment. British Journal of Addiction, 78 , 269-276. doi:10.1111/j.1360-0443.1983.tb02511.x.

Marlatt, G. A. (1990). Cue exposure and relapse prevention in the treatment of addictive behaviors. Addictive Behaviors, 15, 395-399. doi:10.1016/0306-4603(90)90048-3.

Marlatt, G. A. y Gordon, J. (1985). Relapse prevention. Maintenance strategies in the treatment of addictive behaviors. New York: The Guilford Press.

Marlatt, G. A. y Witkiewitz, K. (2005). Relapse prevention for alcohol and drug problems. En: Marlatt, A., Donovan, D. M (Eds), Relapse prevention: maintenance strategies in the treatment of addictive behaviors (2nd Ed), 1-44.

Mons, N. y Beracochea, D. (2016). Behavioral neuroadaptation to alcohol: From glucocorticoids to histone acetylation. Frontiers in Psychiatry, 7, 16. doi:10.3389/ fpsyt.2016.00165.

Oquendo, M., Baca-García, E., Graver, R., Morales, M., Montalvan, V. y Mann, J. (2001). Spanish adaptation of 
the Barratt impulsiveness scale (BIS-11). European Journal of Psychiatry, 15, 147-55.

Patton, J. H., Stanford, M. S. y Barratt, E. S. (1995). Factor structure of the Barratt impulsiveness scale. Journal of Clinical Psychology, 51, 768-774. doi:10.1002/1097-467 9(199511)51:6<768::AID-JCLP2270510607>3.0.CO;2-1.

Rubio, G., Marín, M., Arias, F., López-Trabada, J. R., Iribarren, M. Alfonso, S.,... de Fonseca, F. R. (2018). Inclusion of alcoholic associations into a public treatment programme for alcoholism improves outcomes during the treatment and continuing care period: A 6-year experience. Alcohol and Alcoholism, 32, 1681-1687. doi:10.1093/alcalc/agx078.

Rubio, G., Urosa, B. y Santo Domingo, J. (1998). Validación de la escala de intensidad de la dependencia al alcohol. Psiquiatría Biológica, (Supl 1), 44-47.

Substance Abuse and Mental Health Services Administration. (2011). SAMHSA announces a working definition of "recovery" from mental disorders and substance use disorders. Recuperado de https://store.samhsa.gov/sites/default/files/d7/priv/pep12-recdef.pdf.

Sobell, L. C., Sobell M.B., Leo, G. I. y Cancilla, A. (1988). Reliability of a timeline method: Assessing normal drinkers' reports of recent drinking and a comparative evaluation across several populations. British Journal of Addiction, 83, 393-402. doi:10.1111/j.1360-0443.1988. tb00485.x.

Stautz, K. y Cooper, A. (2013). Impulsivity-related personality traits and adolescent alcohol use: A meta-analytic review. Clinical Psychology Review, 33, 574-592. doi:10.1016/j. cpr.2013.03.003.

Steger, M. F., Frazier, P., Kaler, M. y Oishi, S. (2006). The meaning in life questionnaire: Assessing the presence of and search for meaning in life. Journal of Counseling Psychology, 53, 80-93. doi:10.1037/0022-0167.53.1.80.

Stockwell, T., Murphy, D. y Hodgson, R. (1983). The severity of alcohol dependence questionnaire: Its use, reliability and validity. British Journal of Addiction, 78, 145-155. doi:10.1111/j.1360-0443.1983.tb05502.x.

Tonigan, J. S., McCallion, E. A., Frohe, T. y Pearson, M. R. (2017). Lifetime alcoholics anonymous attendance as a predictor of spiritual gains in the relapse replication and extension project (RREP). Psychology of Addictive Behaviors, 31, 54-60. doi:10.1037/adb0000235.

Ugochukwu, C., Bagot, K. S., Delaloye, S., Pi, S., Vien, L., Garvey, T.,... Ishak, W. W. (2013). The importance of quality of life in patients with alcohol abuse and dependence. Harvard Review of Psychiatry, 21, 1-17. doi:10.1097/ hrp.0b013e31827fd8aa.

Venner, K. L., Matzger, H., Forcehimes, A. A., Moos, R. H., Feldstein, S. W., Willenbring, M. L. y Weisner, C. (2006). Course of recovery from alcoholism. Alcoholism: Clinical and Experimental Research, 30, 1079-1090. doi:10.1111/ j.1530-0277.2006.00121.x.
White, W. L. (2012). Recovery/remission from substance use disorders: An analysis of reported outcomes in 415 scientific reports, 1868-2011 (Drug $\mathcal{E}$ Alcohol findings Review Analysis). Pittsburgh, PA: Philadelphia Department of Behavioral Health and Intellectual Disability Services and the Great Lakes Addiction Technology Transfer Center.

Wilcox, C. E., Pearson, M. R. y Tonigan, J. S. (2015). Effects of long-term aa attendance and spirituality on the course of depressive symptoms in individuals with alcohol use disorder. Psychology of Addictive Behaviors, 29, 382-391. doi: $10.1037 / \mathrm{adb} 0000053$.

Witkiewitz, K., Wilson, A. D., Pearson, M. R., Montes, K. S., Kirouac, M., Roos, C. R.,... Maisto, S. A. (2019). Profiles of recovery from alcohol use disorder at three years following treatment: Can the definition of recovery be extended to include high functioning heavy drinkers? Addiction, 14, 69-80. doi:10.1111/add.14403.

Worley, M. J., Tate, S. R. y Brown, S. A. (2012). Mediational relations between 12-step attendance, depression and substance use in patients with comorbid substance dependence and major depression. Addiction, 107, 19741983. doi:10.1111/j.1360-0443.2012.03943.x. 